Portland State University

PDXScholar

\title{
Posttraumatic Growth Moderates the Effect of Posttraumatic Stress on Quality of Life in U.S. Military Veterans with Life-Threatening Illness or Injury
}

\author{
Erin Martz \\ Rehability and Veterans Affairs Portland Health Care System \\ Hanoch Livneh \\ Portland State University, livnehh@pdx.edu \\ Steven M. Southwick \\ VA Connecticut Healthcare System, West Haven \\ Robert H. Pietrzak \\ Yale University School of Medicine
}

Follow this and additional works at: https://pdxscholar.library.pdx.edu/coun_fac

Part of the Counseling Psychology Commons, and the Counselor Education Commons Let us know how access to this document benefits you.

\footnotetext{
Citation Details

Martz, Erin, Hanoch Livneh, Steven M. Southwick, and Robert H. Pietrzak. "Posttraumatic growth moderates the effect of posttraumatic stress on quality of life in US military veterans with life-threatening illness or injury." Journal of Psychosomatic Research 109 (2018): 1-8.
}

This Article is brought to you for free and open access. It has been accepted for inclusion in Counselor Education Faculty Publications and Presentations by an authorized administrator of PDXScholar. Please contact us if we can make this document more accessible: pdxscholar@pdx.edu. 


\title{
Posttraumatic growth moderates the effect of posttraumatic stress on quality of life in U.S. military veterans with life-threatening illness or injury
}

\author{
Erin Martz ${ }^{\mathrm{a}, *}$, Hanoch Livneh ${ }^{\mathrm{b}}$, Steven M. Southwick ${ }^{\mathrm{c}}$, Robert H. Pietrzak ${ }^{\mathrm{d}}$

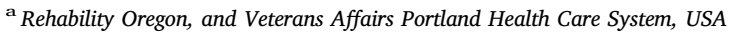 \\ b Rehabilitation Counseling Program, Portland State University, USA \\ ${ }^{\mathrm{c}}$ U.S. Department of Veterans Affairs National Center for Posttraumatic Stress Disorder, VA Connecticut Healthcare System, West Haven, CT, USA \\ ${ }^{\mathrm{d}}$ Department of Psychiatry, Yale University School of Medicine, New Haven, CT, USA
}

\section{Introduction}

Posttraumatic stress disorder (PTSD) has been a focal point of much war-related trauma research $[43,71,87]$. For several decades, a large body of research has examined the relationship between PTSD and physical impairment, disability, and functionality [3,49,50,72]. Wiseman, Foster, and Curtis [88] conducted a review of 41 publications on the topic of traumatic physical injury and mental health. They noted that PTSD was the most frequently researched mental health issue following a traumatic physical injury and that the incidence of PTSD ranged from $30 \%$ to $93 \%$ in individuals who survived a traumatic physical injury. Schweininger and colleagues [69] suggested that "there is something uniquely damaging about high levels of PTSD symptoms in the aftermath of injury" (p. 5). PTSD also has been associated with a lower quality of life (QoL) among war Veterans [68]. In view of the detrimental impact of PTSD on individuals' lives, the present research focused on investigating what factors may increase QoL in the context of PTSD and severe injury or disability among Veterans, in order to promote mental health for this population.

Epidemiological studies on PTSD among Veterans have indicated that not everyone experiencing traumatic events will develop PTSD symptoms. In the National Vietnam Veterans Readjustment Study, Kulka et al. [43] reported that fifteen or more years after their service, $15.2 \%$ of males and $8.1 \%$ of females who were Vietnam theater Veterans had a current diagnosis of PTSD, indicating that about $85 \%$ of the males and $92 \%$ of the females did not have PTSD. These PTSD studies among Veterans and other trauma survivors inevitably led to questions about why people experience PTSD after trauma and how resilience and posttraumatic growth (PTG) may mitigate the experience of PTSD.

PTG refers to positive personal changes that result from the survivor's struggle to cope with trauma and its psychological consequences [78]. PTG is distinct from resilience in that individuals experiencing PTG do not merely return to a pre-trauma baseline, but, in fact, advance to higher than pre-trauma levels in one or more life domains, such as self-perception, relationships with others, greater emotional expressiveness, greater appreciation and enjoyment of life, a more meaningful life, and heightened spirituality $[76,77,79]$. Tedeschi and Calhoun $[10,76,77,79]$ noted that the PTG process does not necessarily decrease emotional distress, because growth after extreme life crises may require an existential struggle for people to rebuild their worldviews. Tedeschi and Calhoun [77] emphasized that "For most trauma survivors, posttraumatic growth and distress will coexist, and the growth emerges from the struggle with coping, not from the trauma itself" (p. 60).

Recent research has explored PTG among Veterans who reported PTSD. Pietrzak and colleagues ([81-83]) conducted a nationally representative study $(N=3157)$ called the National Health and Resilience in Veterans Study (NHRVS), which assessed PTG, resilience, PTSD, and other variables among Veterans. Of all potentially traumatic events, experiencing a life-threatening illness or injury was significantly and positively related to PTG, while experiencing a natural disaster was significantly and negatively related to PTG among the full NHRVS sample that included Veterans exposed to a broad range of potentially traumatic life events [81]. These particular findings from the full NHRVS sample suggested that the relationship between life-threatening illness or injury and PTG warrants a more in-depth examination. This information could help elucidate the role of PTG-both directly and as a potential moderator of illness/injury-related PTSD symptoms-in relation to functioning and QoL in Veterans with life-threatening illness or injury.

\section{Quality of life and chronic illness and disability}

QoL, which is the focus of the present study, has been used as both an outcome variable and a therapeutic goal in literature on coping with life traumas, and more specifically psychosocial adaptation to chronic illness and disability (CID; [5,20,24,45,66]). QoL has been viewed rather consistently as an outcome of these and related psychosocial models of adaptation to CID, trauma, and crisis. QoL is also depicted as a complex, multidimensional, and dynamic construct that incorporates both subjective and objective domains. It reflects a comprehensive

\footnotetext{
* Corresponding author.

E-mail address: rehabilityoregon@gmail.com (E. Martz).
} 
endpoint component in assessing the psychosocial chain of adaptation to life stresses and crises. In a comprehensive review of the literature on QoL and PTSD among war Veterans, Schnurr, Lunney, Bovin and Marx [68] argued that QoL consists of three primary components, including life satisfaction (well-being), functional level, and social-material conditions. Research has also demonstrated that reported levels of QoL after CID are directly influenced by a host of functional, psychosocial, and environmental variables. For example, subjective and objective reports of higher level QoL have been associated with being employed, attaining a higher level of education, having no recent hospitalizations, having no prior substance abuse, perceiving strong emotional and social support, and experiencing lower pain level $[18,23,24,53,54]$ in survivors of SCI and traumatic brain injury (TBI). Surprisingly though, only weak relationships exist between reported levels of post-CID QoL and severity of injury, time since injury [TSI], or medical condition itself $[6,23,24,61]$.

In the context of adaptation to sudden onset of CID, empirical findings have indicated that QoL experienced by severely injured trauma survivors $[19,21,23,24,35]$ was significantly poorer than their QoL prior to injury, or than that reported by the general public. Reported findings also indicated that depressed mood, anxiety, and PTSD $[7,12,18,42,53,74]$ are reliable predictors of lower level of life satisfaction and QoL among cancer, heart disease, SCI, and TBI survivors in both cross-sectional and longitudinal studies. Among individuals who sustained severe traffic-related injuries [85], early reports (1-week post-injury) of depression, anxiety, and PTSD were statistically significant predictors of lower QoL at a later time (6-week post-injury). Similarly, post-injury depression and PTSD were found to be reliable predictors of QoL at 12-month and 18-month follow-up, among individuals who sustained severe bodily injuries [35]. Finally, in a prospective study of Australian trauma-center treated survivors [56], QoL measured at 12-months post-injury was predicted by acute psychological responses, measured immediately following traumatic event (i.e., acute depression, acute and posttraumatic stress disorders).

The above findings have mostly focused on the prediction of QoL from indicators of impairment, disability, and negative psychological states such as depression, anxiety, and PTSD. In contrast, positive psychological resources were found to predict higher perceived QoL, life satisfaction, psychological well-being, and global psychosocial adjustment among SCI survivors ([41,46]; Mortensen et al. [53]). These associations were also found among cancer survivors [14,51], individuals with amputation [25], and individuals with TBI [9]. In a similar vein, PTG has been found to be positively associated with higher QoL among cancer survivors [38], and individuals with TBI [63].

The role of PTG as a buffer or moderator between CID-related or negative affectivity variables (e.g., depression, PTSD) and QoL has been explored by several research teams. PTG was found to moderate posttraumatic stress symptoms on QoL in Morrill et al.' [52] study among women with early stage breast cancer, as well as in Bluvstein et al.'s [7] study among individuals who had experienced coronary heart disease. In a similar vein, Pakenham [57] found that benefit-finding (one of PTG's measures) moderated the relationships between global stress and respondents' affect and life satisfaction in a sample of individuals with multiple sclerosis. Given the need for a better understanding of how to facilitate better QoL in the context of a life-threatening illness or injury, the present research examined the relationships among PTSD symptoms, PTG, and QoL, and the possible moderating effect of PTG on QoL among Veterans.

\section{Aims of study}

The present study has three aims. The first aim is to examine the predictive ability of PTSD and PTG on individuals' QoL among a sample who experienced a range of life-threatening illnesses or injuries. The second aim is to examine whether PTG acts as a protective factor that moderates the effect of PTSD on QoL among this sample with diverse life-threatening illnesses or injuries. The third aim of this study, as an extension of the second aim, is to examine the specific role played by each of the various facets of PTG (i.e., developing more intimate relationships, recognizing new possibilities in one's life, a greater sense of personal strength, greater spiritual development, and a greater appreciation of life) in predicting and moderating QoL among Veterans who have experienced life-threatening illnesses or injuries. In summary, we sought to more carefully examine the multifaceted nature of PTG as a predictor of QoL and as a protective factor that moderates the impact of PTSD on QoL.

\section{Research hypotheses}

1. PTSD symptoms will be negatively associated and PTG positively associated with perceived QoL among Veterans who have experienced life-threatening illnesses and injuries.

2. PTG will moderate the effect of PTSD symptoms on QoL among Veterans who have experienced life-threatening illnesses or injuries, such that Veterans with greater PTG will report greater QoL than Veterans with lower PTG.

3. Each of the five PTG components (subscales) will moderate the effect of PTSD on QoL among Veterans who have experienced lifethreatening illnesses or injuries. However, in the absence of existing clinical or empirical documentation, no specific predictions as to their relative importance were made.

\section{Methods}

\subsection{Participants}

The NHRVS is a nationally representative survey of U.S. Veterans $(N=3157)$. This internet-based survey was conducted from October to December 2011. The present cross-sectional study focused on a subsample of the NHRVS $(N=418)$, which consisted only of individuals who reported a life-threatening illness or injury as their 'worst' traumatic life event. Prior to initiating the proposed analyses, a power analysis was conducted. In order to detect a medium effect size $\left(\mathrm{R}^{2}=0.15\right)$ for linear multiple regression analysis, with alpha $=0.05$, power $=0.95 ; 10-12$ predictors, $N=75$ is suggested (G*Power Statistical Software; [27]), well below the available sample size. The NHRVS study was conducted by using the GfK Knowledge Panel (GfK, Inc., Menlo Park, CA) a probability-based, online survey panel of a nationally representative sample of $>50,000$ U.S. adults that covers approximately $98 \%$ of U.S. households (see [59], for a more detailed descripton and information about post-stratification weights and calibration of the panel). Missing data were handled by multiple imputation using an iterative Markov chain Monte Carlo (MCMC) method, which was used to reduce bias related to item-level missing data $(<5 \%$ across scales). The institutional review board at the Veterans Affairs Connecticut Healthcare System (for the original data collection) and the VA Portland Healthcare System's Research and Development department (for this study's secondary data analysis as a 'science-only study') approved the study's procedures.

On average, the mean age of the subsample of the NHRVS analyzed in this study was $67.2(\mathrm{SD}=11.3$, range $=24-93)$. The majority consisted of men (96.6\%) and white, non-Hispanic (80.7\%), married/ partnered (75.6\%), and retired (62.3\%). Educationally, 33.0\% had received a high school diploma, followed by those who completed some college coursework (21.7\%), Associate's degree (8.6\%), Bachelor's degree (18.8\%), Master's degree (9.9\%), and Professional or Doctorate degrees (3.7\%). The number of lifetime traumas reported by this group ranged from 1 to $14(M=3.8, S D=2.7)$. Compared to the full NHRVS sample, this subsample was older (6.9 years on average, $t$ $(3573)=9.08, p<0.001)$, comprised of a greater percentage of men (6.8\% greater, $\left.\chi^{2}(1)=20.03, \mathrm{p}<0.001\right)$ and retirees $(18.8 \%$ higher, $\left.\chi^{2}(1)=52.56, \mathrm{p}<.001\right)$, and reported a greater mean number of 
traumatic life events $(0.4$ more events, $t(3573)=3.00, p=0.003$. These groups did not differ by race/ethnicity (2.9\% lower in subsample, $\left.\chi^{2}(1)=2.23, \mathrm{p}=0.14\right)$, marital status $(3.0 \%$ lower in subsample, $\left.\chi^{2}(1)=1.95, \mathrm{p}=0.16\right)$, or level of education $\left(\chi^{2}(1)=1.48, \mathrm{p}=0.22\right)$.

\subsection{Measures}

\subsubsection{Trauma history}

The Trauma History Screen (THS; [13]) is a self-report measure that assesses the lifetime occurrence of 14 potentially traumatic events. Events across the lifespan were assessed, including early life events such as physical or sexual assault during childhood, as well as events that more commonly occur in adulthood, such as motor vehicle accident, military combat, and unexpected loss of a close friend or family member. An additional event, life-threatening illness or injury, was added in the NHRVS. Participants who endorsed enduring a lifethreatening illness or injury were asked to list the specific illness or injury that they experienced. The responses were coded into one of 10 categories. The top three life-threatening illnesses or injuries that were reported were: Sudden-onset condition (heart attack or stroke; $40 \%$ ), cancer (23\%), and infectious disease/infection (8.6\%).

\subsubsection{Medical conditions}

All respondents in this subsample were asked whether they have ever been diagnosed by a physician or other health professional during the past 12 months with one or more of 17 medical conditions, including diabetes mellitus, cardiovascular disease, stroke, cirrhosis or any other form of liver disease, gastrointestinal disease, arthritis, or sexually transmitted diseases (see [60] for further details). Of note, these medical conditions and the ones identified by respondents as their 'worst' traumatic event on the THS were not necessarily mutually exclusive.

\subsubsection{PTSD}

PTSD was measured by the PTSD Checklist-Specific Stressor (PCL-S; [86]). The PCL-S contains 17 items that functions as screening instrument based on the Diagnostic and Statistical Manual for Mental Disorders-IV [2]. Items on the PCL-S were scored on a five-point scale, ranging from 0 ("Not at all") to 4 ("Extremely"). Cronbach's alpha coefficient in the present sample was 0.95 . Total scores range from 17 to 85, with higher scores reflecting greater severity of PTSD symptoms. The mean (M) and standard deviation (SD) for this sample was $M=24.96$ and $S D=11.96$.

\subsection{4. $P T G$}

PTG was measured using the Posttraumatic Growth Inventory-Short Form (PTGI-SF; [11]). The PTGI-SF contains 10 items that assess positive changes resulting from a traumatic event. Internal reliability (Cronbach's alpha) for the PTGI-SF Total was reported as 0.89 (0.94 in the present sample). The PTGI-SF assesses five factors of PTG (2 items per factor) and their Cronbach's alpha coefficients in the original study, and the present sample, respectively were: relating to others (RO; 0.86; 0.86), new possibilities (NP; 0.82; 0.84), personal strength (PG; 0.78; 0.87 ), spiritual change (SC; $0.80 ; 0.92)$, and appreciation of life (AL; $0.77 ; 0.81$ ). Items were scored on a 6 -point scale, from $0=$ "I did not experience this change as a result of my crisis" to $5=$ "I experienced this change to a very great degree as a result of my crisis." Total scores per subscale range from 0 to 10 , and from 0 to 50 for the PTGI-Total, with higher scores reflecting greater posttraumatic growth [11]. The means and standard deviations for this sample were the following: PTGI total $(M=20.86, S D=14.865)$; PTGI New Possibilities $(M=3.84$, $S D=3.29)$; PTGI Personal Strength $(M=4.19, S D=3.19)$; PTGI Spiritual Change $(M=3.92, S D=3.63)$; PTGI Appreciation of Life $(M=4.75, S D=3.22)$; PTGI Relationships with Others $(M=3.97$, $S D=3.22$ ).

\subsubsection{Disability in activities of daily living}

Disabilities in activities of daily living and instrumental activities of daily living were assessed with the following questions: ADL disabilities: "At the present time, do you need help from another person to do the following?" (bathe; walk around your home or apartment; get in and out of chair; [30]); and IADL disabilities: "At the present time, do you need help form another person to do the following?: (shop for groceries, clothing or other items; go to the doctor; travel to visit friends, go to church or temple, etc.; pay bills or manage money; prepare meals; do household chores; take medication properly).

\subsubsection{Quality of life}

The outcome variable in this study was measured using the Quality of Life Enjoyment and Satisfaction Questionnaire-Short Form (Q-LES-QSF; [26]). It contains 14 items, which measure individuals' satisfaction in the past week with various aspects of their lives (e.g., physical health, mood, household activities, social relationships, leisure activities). Items are scored on a 5-point scale that ranges from $1=$ very poor to $5=$ very good. In the original validation study [26], Q-LES-Q-SF scores were found to be reliable and valid measures of degree of enjoyment and satisfaction in various areas of daily functioning; further, Q-LES-QSF scores were related to, but not redundant, with measures of illness severity and depressive symptoms. Cronbach's alpha coefficient in the present sample was 0.95 . Total scores range from 14 to 70 . Higher scores reflect better quality of life. The mean and standard deviation for this sample was $M=52.87$ and $S D=10.98$.

\subsection{Data analysis}

Prior to undertaking the correlational and multiple regression analyses, the variables pertinent to this study were examined for their compliance with assumptions of normality and linearity, as well as for existence of multicollinearity. With regards to skewness, none of the values of the inspected variables fell outside the range of -1.03 to +2.6 (most measured between \pm 1.00 ), thus suggesting only minimal and positive level of skewness. Inspection of variable kurtosis values indicated that all but one variable (PTSD symptoms) were well within the recommended limits (ranging from -0.92 to -1.30 ). The kurtosis $Z$-score for PTSD, 7.13, suggested a peaked but centered distribution [74]. None of the measures of multicollinearity indicated deviation from traditional accepted tolerance values [58]. To evaluate Hypothesis 1 , we computed zero-order correlations between the seven demographic and clinical control variables, PTSD, the five PTGI subscales, and QoL. To evaluate Hypothesis 2, we conducted a multiple linear regression analysis with interaction procedures advocated by Baron and Kenny [4] and Holmbeck [36] was used. Prior to undertaking the moderator analysis, the predictor variable (PTSD), PTG-Total, and each of the five potential moderator variables (each PTG component) were centered. Then interaction terms were constructed by multiplying the centered PTSD by each centered PTG component. The centering procedure serves to reduce the threat of multi-collinearity problems. In order to answer hypotheses two and three, the following blocks of variables were entered in the multiple regression analysis (MRA) and were regressed on the criterion variable (QoL): the eight control variables (block 1), the predictor (PTSD; block 2), the moderator (PTG; block 3), and the interaction variable of PTSD and PTG (block 4). Given that PTG-Total (2nd hypothesis) and the five PTG factors (3rd hypothesis) were analyzed, there were a total of 6 separate MRAs that were run, each of which had different entries in blocks 3 and 4. Finally, to evaluate Hypothesis 3, (i.e., the moderation of PTG subscales), the five interaction terms (separately), reflecting the products of the five main effects (e.g., PTSD $\times$ New Possibilities) were entered, separately, in block 4 of the regression model. 


\section{Results}

The following continuous control variables were significantly correlated with QoL: 1) Age, $r=0.314$, p $<0.001$; 2) sum of number of lifetime traumas, $r=-0.405 \mathrm{p}<0.001$; 3) sum of disabilities in activities of daily living (ADL): $r=-0.358 \mathrm{p}<0.001$; 4) sum of disabilities in instrumental activities of daily living (IADL), $r=-0.571$ $\mathrm{p}<0.001$; and 5) household income, $r=-0.219$, $\mathrm{p}<0.001$. TSI was not significantly correlated with QoL, $r=0.015 \mathrm{p}=0.761$. The following categorical control variables were significantly correlated with QoL scores: 1) Ethnicity, $\mathrm{p}<0.001$, with higher QoL scores among Whites (non-Hispanics); and 2) marital status, $\mathrm{p}<0.001$, with higher scores among the married group. Combat vs. noncombat veteran, education, and gender did not exhibit significant correlations with QoL.

Hypothesis 1. The first hypothesis of this study addressed the association between PTSD and QoL and PTG-Total and QoL, and was partially supported. Both variables (PTSD, PTG-Total) were significantly and negatively associated with perceived QoL ( $r=-0.622, \quad \mathrm{p}<0.001 ; \quad r=-0.135, \mathrm{p}<0.001$, respectively). While the inverse correlation between QoL and PTSD was anticipated, the inverse correlation between QoL and PTG-Total was unexpected, although infrequently reported in the literature (e.g., [7]). Table 1 shows zero-order correlations among QoL, PTSD, and the five PTG subscales. It is noteworthy that all five PTG components also exhibited inverse associations with QoL. More specifically, the correlations between QoL and the five PTG subscales ranged from -0.05 to -0.21 (three of which were statistically significant). The experience of PTSD was also associated with each of the PTG subscales, ranging from 0.27 to 0.38 (all statistically significant).

Hypothesis 2. The second hypothesis focused on the role that PTGTotal may have in moderating the impact of PTSD symptoms on QoL. The second hypothesis was supported in its entirety. The interaction of PTSD and PTG-Total added significantly to the total variance found in QOL (see Table 2). After the entry of the control variables in block 1, PTSD was added to the model in block 2 and was found to be significant $\left(\Delta \mathrm{R}^{2}=0.052, \Delta \mathrm{F}(1,350)=37.54, \mathrm{p}<0.001 ; \mathrm{f}^{2}=0.096\right.$, small to medium ES). In block 3, PTG-Total was added to the model and was found to be significant $\left(\Delta \mathrm{R}^{2}=0.013, \Delta \mathrm{F}(1,349)=9.91, \mathrm{p}=0.002\right.$; $\mathrm{f}^{2}=0.029$, small ES). Finally, in block 4 , the interaction variable of PTSD $\times$ PTG-Total was added to the model and was found to be significant $\quad\left(\Delta \mathrm{R}^{2}=0.020, \quad \Delta \mathrm{F} \quad(1, \quad 348)=15.002, \quad \mathrm{p}<0.001\right.$; $\mathrm{f}^{2}=0.04$, small ES).

The reversed direction of correlational coefficient values, between PTG and QoL, from their zero-order (see Table 1) to Block III multiple regression analysis (MRA) values (see Table 2), is most likely due to the operation of suppression effects periodically found in hierarchical MRAs. In this particular case, the introduction of PTSD scores in the second MRA block, scores which were negatively and significantly correlated with the outcome scores of QoL, acted to reverse the PTG-
Table 2

Multiple regression analysis (MRA) models of PTSD, PTG (and its components), and interactions on QOL.

\begin{tabular}{|c|c|c|c|c|c|c|}
\hline Regression model & $\mathrm{R}$ & $\mathrm{R}^{2}$ & $\mathrm{~F} \Delta$ & $\beta$ & $\mathrm{t}$ & $\mathrm{p}$ \\
\hline Block 1 & 0.677 & 0.458 & 42.423 & & & \\
\hline (Constant) & & & & - & 11.119 & $<0.001$ \\
\hline Age & & & & 0.146 & 3.231 & 0.001 \\
\hline $\begin{array}{l}\text { Number lifetime } \\
\text { traumas }\end{array}$ & & & & -0.197 & -4.52 & $<0.001$ \\
\hline ADL disabilities & & & & -0.086 & -1.65 & $<0.001$ \\
\hline IADL disabilities & & & & -0.393 & -6.812 & $<0.001$ \\
\hline Race/ethnicity & & & & -0.004 & -0.091 & 0.927 \\
\hline Marital status & & & & -0.051 & -1.191 & 0.234 \\
\hline Household income & & & & 0.217 & 5.267 & $<0.001$ \\
\hline Block 2 & 0.714 & 0.510 & 37.541 & & & \\
\hline PTSD-past month & & & & -0.352 & -6.127 & $<0.001$ \\
\hline \multicolumn{7}{|l|}{ Block 3 (Separate } \\
\hline PTG-total & 0.724 & 0.524 & 9.911 & 0.126 & 3.148 & 0.002 \\
\hline PTG-relating to others & 0.730 & 0.533 & 17.187 & 0.160 & 4.146 & $<0.001$ \\
\hline PTG-new possibilities & 0.724 & 0.524 & 10.078 & 0.128 & 3.175 & 0.002 \\
\hline PTG-personal strength & 0.722 & 0.521 & 7.690 & 0.108 & 2.773 & 0.006 \\
\hline $\begin{array}{l}\text { PTG-spiritual } \\
\text { development }\end{array}$ & 0.720 & 0.518 & 5.606 & 0.094 & 2.368 & 0.018 \\
\hline $\begin{array}{l}\text { PTG-appreciate life } \\
\text { Block } 4 \text { (interactions as } \\
\text { separate MRAs) }\end{array}$ & 0.716 & 0.513 & 2.085 & 0.059 & 1.444 & 0.15 \\
\hline PTSD $\times$ PTG-total & 0.737 & 0.543 & 15.002 & 0.195 & 3.873 & $<0.001$ \\
\hline $\begin{array}{l}\text { PTSD } \times \text { PTG-relating to } \\
\quad \text { others }\end{array}$ & 0.742 & 0.550 & 13.163 & 0.165 & 3.628 & $<0.001$ \\
\hline $\begin{array}{c}\text { PTSD } \times \text { PTG-new } \\
\text { possibilities }\end{array}$ & 0.734 & 0.539 & 11.239 & 0.172 & 3.353 & 0.001 \\
\hline $\begin{array}{l}\text { PTSD } \times \text { PTG-personal } \\
\text { strength }\end{array}$ & 0.738 & 0.545 & 18.721 & 0.191 & 4.327 & $<0.001$ \\
\hline $\begin{array}{c}\text { PTSD } \times \text { PTG-spiritual } \\
\text { development }\end{array}$ & 0.727 & 0.528 & 7.771 & 0.136 & 2.788 & 0.006 \\
\hline $\begin{array}{l}\text { PTSD } \times \text { PTG-appreciate } \\
\quad \text { life }\end{array}$ & 0.727 & 0.529 & 12.030 & 0.189 & 3.468 & 0.001 \\
\hline
\end{tabular}

Note. $\quad \mathrm{MRA}=$ multiple regression analysis; $\mathrm{ADL}=$ activities of daily living; IADL = instrumental activities of daily living; PTSD = posttraumatic stress disorder; PTG $=$ posttraumatic growth.

QoL correlations from negative, but low, (Table 1$)$ to positive ( $\beta$ values in Block III).

As can be gleaned from the standardized scores in Fig. 1, under conditions of low PTSD ( $-1 \mathrm{SD}$ ), the two PTG-Total groups (high vs. low) did not differ as to their reported QoL; whereas under conditions of high PTSD ( $+1 \mathrm{SD})$, the two groups evidenced significant differences, such that those who experienced high PTG (vs. low PTG) reported higher QoL.

Hypothesis 3. The moderator hypothesis was supported in its entirety because the all five of the interaction terms of block 4 were significant (see Table 2). The interaction of PTSD and each of the five PTG components added significantly to the total variance found in QoL. More specifically, in blocks 3a through 3e (separately for each of the

Table 1

Zero-order correlations among QOL, PTSD-Total, and five PTG factors.

\begin{tabular}{|c|c|c|c|c|c|c|c|}
\hline & QOL & $\begin{array}{l}\text { PTSD past } \\
\text { month }\end{array}$ & $\begin{array}{l}\text { PTG relating to } \\
\text { others }\end{array}$ & PTG New possibilities & $\begin{array}{l}\text { PTG personal } \\
\text { strength }\end{array}$ & $\begin{array}{l}\text { PTG spiritual } \\
\text { development }\end{array}$ & PTG appreciate life \\
\hline QOL & 1 & & & & & & \\
\hline PTSD past month & $-0.622^{* *}$ & 1 & & & & & \\
\hline PTG relating to others & -0.050 & $0.289^{* * *}$ & 1 & & & & \\
\hline PTG new possibilities & $-0.137^{* *}$ & $0.379^{* *}$ & $0.765^{* *}$ & 1 & & & \\
\hline PTG personal strength & -0.070 & $0.272^{* * *}$ & $0.812^{* * *}$ & $0.783^{* *}$ & 1 & & \\
\hline PTG spiritual development & $-0.137^{* *}$ & $0.329^{* *}$ & $0.798^{* *}$ & $0.819^{* *}$ & $0.755^{* *}$ & 1 & \\
\hline PTG appreciate life & $-0.208^{* *}$ & $0.364^{* * *}$ & $0.687^{* * *}$ & $0.784^{* *}$ & $0.700^{* *}$ & $0.674^{* *}$ & 1 \\
\hline
\end{tabular}

Note: All correlates with QOL were centered. QOL = quality of life; PTSD = posttraumatic stress disorder; PTG = posttraumatic growth.

** Correlation is significant at the 0.01 level (2-tailed). 


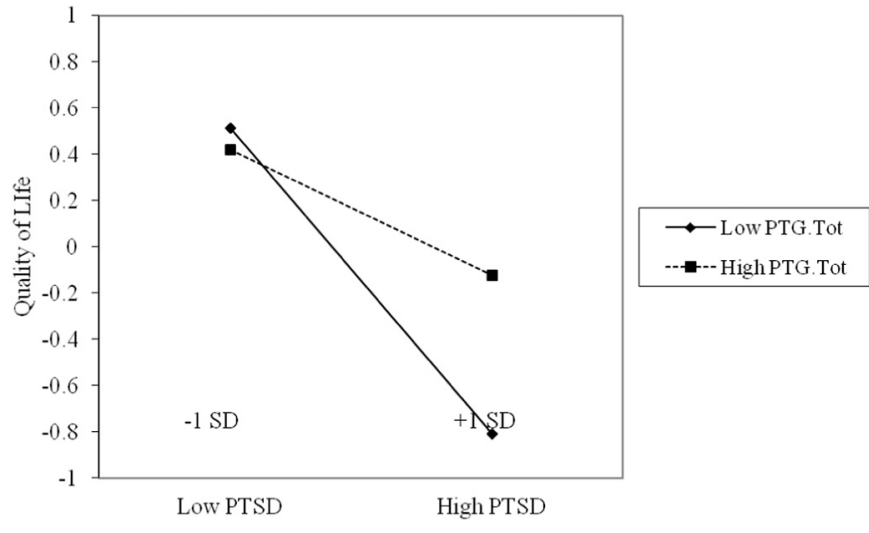

Fig. 1. Interactions of PTSD-past month and PTG-total on QOL.

PTG components), each of these components was tested for its unique contribution to the variance in QoL. As can be seen from Table 2, four of the five PTG components contributed significantly to the variance (except appreciation of life). The size of these individually significant contributions ranged from $\Delta R^{2}=0.008, \Delta F(1,349)=5.61, \beta=0.09$, $\mathrm{p}=0.018 \quad$ (spiritual development) to $\Delta \mathrm{R}^{2}=0.023, \Delta \mathrm{F} \quad(1$, $349)=17.19, \beta=0.15, p<0.001$ (relations to others). Effect sizes for these PTG components ranged from $\mathrm{f}^{2}=0.016$ to $\mathrm{f}^{2}=0.047$ (small ESs).

Finally, in blocks 4a through 4e (separately for interaction term of PTSD $\times$ PTG component), each of these products was tested for its unique contribution to the variance in QoL. As Table 2 reveals, all five interaction products (PTSD $\times$ PTG component) added significantly to the variance in QoL, beyond the combined contributions of the earlier three blocks of variables. These interaction values ranged in size from $\Delta \mathrm{R}^{2}=0.011, \Delta \mathrm{F}(1,348)=7.71, \beta=0.136, \mathrm{p}=0.006$ (spiritual development; $\mathrm{f}^{2}=0.023$, small ES) to $\Delta \mathrm{R}^{2}=0.024, \Delta \mathrm{F}(1,348)=18.72$, $\beta=0.191, p<0.001$ (personal strength; $f^{2}=0.05$, small ES). The same patterns that were evident in the PTSD $\times$ PTG-Total interaction (see Fig. 1) emerged with only minor deviations for each of the five PTG components; exhibiting these graphs is, therefore, not included here [these graphs are available from the first author upon request].

\section{Discussion}

The aims of this research were to investigate whether PTSD and PTG influenced individuals' QoL, whether PTG moderated the hypothesized association between PTSD and QoL, and whether all five PTG factors moderated the PTSD and QoL association. The results of this crosssectional study indicated, not surprisingly, that PTSD was inversely associated with QoL. The finding that PTG was associated positively with QoL in the fully adjusted models is consistent with prior work. In research with a larger sample containing Veterans with a broad range of traumatic life events, it was found that among Veterans with PTSD, those with PTG reported better mental functioning and general health than those without PTG [81]. Further, the results suggested that PTG (PTGI-Total and all five PTGI subscales) exerts both a direct influence, as well as a moderated effect, on individuals perceived QoL, after controlling for the influence of sociodemographic and clinical variables, indicating that under conditions of higher experienced PTSD, PTG has a larger influence on QoL than under lower reported PTSD.

\subsection{PTG and quality of life}

As previously mentioned, PTG levels (PTGI-Total and its 5 subscales) were significantly and negatively associated with levels of perceived QoL, but PTG was a significant, positive predictor of QoL in the MRA in this study. The complex nature of the link between measures of
QoL and PTG was documented in a study of survivors of breast cancer [44], where a curvilinear relationship between these two measures was detected, thus masking any efforts to examine linear relationships between QoL and PTG (benefit-finding) and suggesting that under conditions of both low and high PTG participants reported higher level of QoL, while under moderate degree of PTG, lower levels of QoL were reported.

Further, both PTGI-Total and all five PTGI subscales successfully moderated the influence of PTSD on perceived QoL (Hypotheses 2 and 3). More specifically, under conditions of higher experienced PTSD, the moderating role of each of the five PTGI subscales, as well as that of the total score, assumed a larger influence on Veterans' perceived QoL than under lower reported PTSD. This finding receives some support in empirical research among individuals with cancer [44,70]. For example, in a sample of women with early-stage breast cancer, Sears et al. [70] reported that more intensely experienced stress (e.g., thought intrusion, avoidance) was related to increased posttraumatic growth 12 months following diagnosis. The latter, in turn, was predictive of positive mood and perceived health, both indicators of QoL.

While several studies $[7,52,57]$ reported similar findings to these of the present study, other researchers have not found such associations. For example, among TBI survivors, Hawley and Joseph [31] found that respondents who reported higher levels of anxiety and depression, at a 10-year follow-up, experienced lower levels of positive changes in outlook (a measure of PTG). The majority of the respondents, however, demonstrated positive growth over the 10-year follow-up of the study. This increased growth was positively associated with better adjustment, as measured by their overall community integration, thus suggesting that anxiety had a direct effect on adjustment that was not moderated by growth. The lack of consistent findings in the literature on the effect of PTSD and PTG interaction on QoL may reflect a variety of other, often untested yet interacting influences, such as degree of CID severity, the impact of life-threatening conditions, the nature of the traumatic event, success in employing adaptive coping strategies and resources in the past and the present, the effect of certain lifestyle practices (e.g., use of alcohol and drugs), and the influence of personality characteristics such as resilience, optimism and hope. There is, however, an additional factor that must be considered, namely the veracity of the reported PTG, or as often posited in the literature: Is PTG reflective of true psychological growth or is it a derivative of denial-like, positive illusions $[47,75,77]$ ?

Complicating matters further, PTG levels can fluctuate over time. For example, a recent study using the full NHRVS sample revealed five different longitudinal courses of PTG over a 2-year period-Consistently Low (33.6\%), Moderately Declining (19.4\%), Increasing PTG (16.8\%), Dramatically Declining (15.7\%), and Consistently High (14.5\%); of note, more than half (59.4\%) of Veterans who reported at least "moderate" PTG at baseline maintained that level of PTG 2 years later [83]. Nevertheless, greater PTG in relation to one's worst trauma-specifically, Personal Strength-has been linked to greater resilience to subsequent traumas, as assessed using measures of the severity and incidence of PTSD [82]. Thus, it appears that despite having a relatively heterogeneous course over time, PTG can foster resilience to subsequent traumas. Additional longitudinal research is needed to disentangle directional associations between PTSD, PTG, and QoL in trauma survivors, as well as mediators and moderators of these associations.

With regard to future research concerning QoL, as well as its closely aligned constructs of subjective well-being and life satisfaction, both the generic psychology (e.g., [22,34]) and the more specific CID-linked (e.g., $[6,56]$ ) literatures consistently indicate that these constructs are multidimensional in nature, and do not necessarily reflect a single, unified picture. The contributing domains to QoL have been hypothesized to either be parallel in nature, that is unique yet somewhat overlapping, or concentric (i.e., nested) in structure. Regardless of QoL's empirical structure (see Introduction), there are several domains which 
have been frequently recognized in the literature, that is: (a) the subjective (well-being, life satisfaction, positive and negative affect) domain; (b) the health, medical, and functional domain; (c) the socialinterpersonal domain; and (d) the materialistic-economic domain. The partial independence of these domains suggest that they may be differentially influenced by PTG, and therefore, uniquely predicted by the various components of PTG. Future research, then, would benefit from studying in more detail how different aspects of QoL (e.g., subjective vs. objective, psychological vs. medical-functional, cognitive-affective vs. materialistic-economic) are predicted by the various components that make up PTG. Indeed, if PTG is more reflective of subjective levels of either genuine or illusory growth following traumatic events such as CID onset, it could be argued that it would be a more robust predictor of the subjective and cognitive-affective aspects of QoL, rather than its objective, functional, and materialistic ones.

\subsection{PTG and PTSD}

A few unexpected findings deserve further explanation. The positive relationship between PTG and PTSD (see the section on Hypothesis 1) in this sample of Veterans with life-threatening injuries and health conditions, although not hypothesized by us, have been observed in other clinical populations, such as cancer, heart disease, and TBI survivors $[7,62,80]$. These studies, as well as the earlier theorizing of several stress and trauma authors, including Janoff-Bulman [37] and Tedeschi and Calhoun [77], support the coexistence of PTG and distress, in which growth emerges from, but does not necessarily eliminate distress. Further, Maercker and Zoellner [47] proposed a possible dual nature of PTG, containing a constructive side to PTG that involves reappraisal of the trauma, and an illusory or distractive side to PTG that involves a "palliation" or alleviation of emotional reactions. These theoretical approaches suggest that the very essence of PTG indicates an unfolding process whereby the individual copes with the presence of traumatic stress over time while rebuilding further resiliency and positive psychosocial adaptation.

Another reason that may explain why there was a positive relationship between PTG and PTSD in the present study is the issue of time. Tedeschi and colleagues [77,78] argue that either differential time frames (i.e., early vs. late during the coping with trauma process) or the elasticity of the psyche following severe traumas permit for both positive and negative affect to coexist during the coping process. Accordingly, the link between PTG and PTSD possibly was confounded by the overlap among the measurement of time since initial injury, given that the recognition of a traumatic event, realization of disability, and onset of chronic functional limitations have been found to be often moderated by the passage of time since trauma onset in other studies $[17,29,31]$. Tedeschi and Calhoun [76,77] suggested that early on in the coping with trauma process, automatic, unconscious, intrusive cognitive reactions are the hallmark of PTG, thus permitting denial-like reactions to be prevalent and usher in positive illusory beliefs of growth. Later on, more deliberate, conscious, reflective cognitive processes may predominate, thus permitting inherent resilience and strength-based growth experiences to gradually navigate adaptation toward a positive outcome after experiencing trauma. Future research is needed to elucidate other factors that may mediate and moderate the relation between PTSD, PTG, and QoL, including illness-related factors such as the nature and severity of illness/injury and time since injury, as well as psychosocial factors such as psychological resilience, purpose in life, and social support.

In view of the empirical observations on the co-existence of PTG and PTSD in this study and the greater impact of PTG in the context of higher PTSD, healthcare professionals should seek information on their clients' PTG perspectives after trauma. Further, because growth after trauma does not necessarily eliminate distress, clinicians must be cognizant that PTG should be assessed along with (not instead of) indicators of PTSD or other reports of distress. Individuals' experience of
PTG after trauma may signal that they, despite struggling existentially, are seeking new forms of meaning in their lives following exposure to traumatic events. In view of the results of this study that PTG moderates the impact of PTSD on QoL, these attempts to process and reframe the trauma should be encouraged, even if individuals' levels of distress remain elevated.

\subsection{Implications for healthcare professionals}

Healthcare professionals who serve individuals with life-threatening injuries and medical conditions may attempt to assist these individuals with finding some positive aspects of life that can instill new meanings, despite the fact that their medical conditions may never be cured and could even worsen over time. Psychological interventions using cognitive-behavioral principles, such as acceptance and commitment therapy (ACT; $[32,33]$ ), can be helpful in reducing the resistance to and avoidance of distressing thoughts and emotions related to stressful health conditions by using mindfulness and by setting goals that are meaningful to the individual. Research is emerging about the effectiveness of ACT in treating a range of mental and physical health problems [1], and for promoting health-behavior change [89].

Another psychological intervention, Coping Effectiveness Training $[15,16]$ is useful for individuals with a range of chronic health conditions and illnesses [40,55]. CET teaches a framework for appraising stressors, recognizing their changeable and unchangeable aspects, learning new coping skills, and selecting the coping strategy that is most appropriate to the type of stress that is experienced (e.g., using problem-focused strategies for changeable situations, and emotion-focused coping for unchangeable situations). In addition, much of the literature generated by rehabilitation counseling and rehabilitation psychology has enumerated approaches and techniques that help individuals with health conditions and disabilities seek employment and/ or community reintegration after the onset of a CID, which, in turn, can provide further sources of meaning and benefit-finding for these individuals $[28,39,48]$.

The results of the present study suggest that PTG is an important specific target for clinicians who are assisting individuals with medical conditions. More specifically, the present study's finding, that PTG moderates the impact of PTSD on QoL, suggests that therapeutic efforts to enhance PTG might help to reduce the negative effects of PTSD symptoms among Veterans who have experienced life-threatening illness or injury, particularly Veterans with a high level of PTSD symptoms. In clinical settings, individuals with PTSD generally receive treatments designed to reduce negative symptoms. However, it may also be possible to help individuals with PTSD by employing interventions specifically designed to foster PTG, given that PTG can attenuate the influence of PTSD symptoms on individuals' overall QoL. The following paragraphs include suggestions of possible interventions, and which may promote PTG and which are based on findings from recent research studies.

Of particular relevance to the current study, Tedeschi and McNally [78] have recommended that 5 elements be included in therapeutic enhancement strategies designed to foster PTG in combat Veterans. These include: (1) understanding trauma response as a precursor to PTG, (2) emotion-regulation enhancement, (3) constructive self-disclosure, (4) creating a trauma narrative with PTG domains, and (5) developing life principles that are robust to challenges. Although most traditional psychotherapies for PTSD do not specifically target PTG, PTG has been measured in response to some of these therapies. For example, Roepke [67] conducted a meta-analysis of PTG and traditional psychosocial interventions for PTSD. In 11 of the 12 studies examined by Roepke, traditional therapeutic interventions (e.g., cognitive-behavioral therapy or written or verbal self-expression/disclosure interventions for PTSD) were associated with gains in PTG, with five of the 12 studies showing significant improvements in PTG as compared to control groups. In one of the few PTSD RCTs that specifically targeted 
PTG, Wagner et al. [84] provided couples with 15-sessions of cognitive behavioral conjoint therapy (CBCT) for PTSD, with one session directly addressing PTG. The researchers found that the group receiving the intervention, compared to the waitlist group, exhibited a significant increase in PTG and concluded that having both supportive conjoint therapy and an explicit focus on PTG may help to foster growth among trauma survivors. Another possible resource is Ramos, Leal, and Tedeschi's [65] group psychotherapy protocol for facilitating PTG among individuals with non-metastatic breast cancer. In a RCT, Ramos et al. [64] reported to be effective in increasing PTG among women with breast cancer.

Because PTG is typically considered to be within the realm of positive psychology, interventions based on positive psychology should also be considered as techniques to facilitate PTG. For example, Bolier et al.' [8] meta-analysis of interventions based on positive psychology indicated that small but significant effects on subjective well-being, psychological well-being, and depression, with the first two effects were sustained over a 3-6-month follow-up period. In summary, further research is needed to evaluate the efficacy of interventions particularly designed to foster PTG, as well as to investigate how and to what extent other therapies, many of which are considered within the realm of positive psychology, may foster PTG among Veterans and civilians affected by life-threatening illness or injury.

\subsection{Limitations}

The findings of this study should be viewed within the context of several limitations. First, the sample was comprised of mostly white, older, married, male Veterans. Therefore, these findings cannot be generalized to more heterogeneous groups of people with CID. Second, data used for analysis in this study were obtained exclusively by means of self-report measures, and are therefore subject to reactive confounds, including social desirability, defensiveness, cognitive capacity to process and complete a large number of written forms, and at times memory deficits (e.g., a correlate of TBI and other neurological conditions). Third, the findings reported in this study are derived from various correlational analyses of available data. Therefore, no causative relationships among the variables examined in this study should be assumed in interpreting the findings. For example, it could be argued that one's perceived QoL exerts a reliable effect on the various PTG indicators, and therefore could have been studied as a moderator in predicting PTG. Fourth, the 10-item PTGI-SF measure employed in this study, despite its obvious merits, may nevertheless preclude understanding of how specific dimensions of PTG, directly and interactively with PTSD, relate to QoL. Fifth, the QoL measure in this study is broad in nature and reflects overall quality of life enjoyment and satisfaction and does not differentiate between specific domains of quality of life, which may be uniquely related to PTSD, PTG, and their interaction. Finally, PTG explained a rather modest $2-3 \%$ of the added variance in QoL and the added variance contributed by the interaction of PTSD and PTG was of a similar magnitude. Such values, however, are not uncommonly derived from MRA-based studies and reported in the fields of psychological and social sciences.

\section{Conclusion}

Notwithstanding these limitations, results of this study suggest that PTG moderates the relation between PTSD symptoms and QoL in U.S. Veterans who reported a life-threatening illness or injury as their 'worst' traumatic event. While severity of illness/injury-related PTSD symptoms were strongly and negatively related to QoL, greater perceptions of PTG, particularly relating to others, new possibilities, and personal strength, were positively related to QoL and moderated the effect of PTSD symptom severity on QoL, predominantly among Veterans who reported a high level of PTSD symptoms. Taken together, these results underscore the importance of interventions designed to reduce PTSD symptoms and to bolster PTG in Veterans who experienced a lifethreatening illness or injury. Further research is needed to evaluate prospective interrelationships between PTSD symptoms and PTG over time, and to evaluate the efficacy of interventions that target both PTSD and PTG in promoting QoL and related outcomes in this population.

\section{Conflicts of interest}

There are no conflicts of interests to report.

\section{Disclaimer}

The views expressed in this article are those of the authors and do not necessarily reflect the position or policy of the Department of Veterans Affairs or the United States government.

\section{References}

[1] J.G. A-tjak, M.L. Davis, N. Morina, M.B. Powers, J.A. Smits, P.M. Emmelkamp, A meta-analysis of the efficacy of acceptance and commitment therapy for clinically relevant mental and physical health problems, Psychother. Psychosom. 84 (1) (2015) 30-36.

[2] American Psychiatric Association, Diagnostic and statistical manual of mental disorders, $4^{\text {th }}$ edition (DSM-IV), American Psychiatric Association, 1994.

[3] M.L. Anderson, D.M. Ziedonis, L.M. Najavits, Posttraumatic stress disorder and substance use disorder comorbidity among individuals with physical disabilities: findings from the National Comorbidity Survey Replication, J. Trauma. Stress. 27 (2) (2014) 182-191.

[4] R.M. Baron, D.A. Kenny, The moderator-mediator variable distinction in social psychological research: conceptual, strategic, and statistical considerations, J. Pers. Soc. Psychol. 51 (6) (1986) 1173-1182.

[5] M. Bishop, Quality of life and psychosocial adaptation to chronic illness and acquired disability: a conceptual and theoretical synthesis, J. Rehabil. 71 (2) (2005) 5-13.

[6] M. Bishop, S.M. Smedema, E.J. Lee, E. Da Silva Cardoso, Outcome measurement and adjustment to chronic illness and disability: Quality of life, in: F. Chan, J. Chronister (Eds.), Understanding Psychosocial Adjustment to Chronic Illness and Disability: A Handbook for Evidence-Based Practitioners in Rehabilitation, Springer, N.Y., 2009, pp. 521-558.

[7] I. Bluvstein, L. Moravchick, D. Sheps, S. Schreiber, M. Bloch, Posttraumatic growth, posttraumatic stress symptoms, and mental health among coronary heart disease survivors, J. Clin. Psychol. Med. Settings 20 (2013) 164-172.

[8] L. Bolier, M. Haverman, G.J. Westerhof, H. Riper, F. Smit, E. Bohlmeijer, Positive psychology interventions: a meta-analysis of randomized controlled studies, BMC Public Health 13 (119) (2013) 1-20.

[9] I. Brands, S. Köhler, S. Stapert, D. Wade, C. van Heugten, Influence of self-efficacy and coping on quality of life and social participation after acquired brain injury: a 1 year follow-up study, Arch. Phys. Med. Rehabil. 95 (12) (2014) 2327-2334.

[10] L.G. Calhoun, R.G. Tedeschi (Eds.), Handbook of Posttraumatic Growth: Research and Practice, Routledge, 2014.

[11] A. Cann, L.G. Calhoun, R.G. Tedeschi, K. Taku, T. Vishnevsky, K.N. Triplett, S.C. Danhauer, A short form of the posttraumatic growth inventory, Anxiety Stress Coping 23 (2) (2010) 127-137.

[12] I. Carboon, V.A. Anderson, A. Pollard, J. Szer, J.F. Seymour, Posttraumatic growth following a cancer diagnosis: do world assumptions contribute? Traumatology 11 (4) (2005) 269-283.

[13] E.B. Carlson, S.R. Smith, P.A. Palmieri, C.J. Dalenberg, J.I. Ruzek, R. Kimerling, T.A. Burling, D.A. Spain, Development and validation of a brief self-report measure of trauma exposure: the trauma history screen (PDF), Psychol. Assess. 23 (2011) 463-477.

[14] C.S. Carver, R.G. Smith, M.H. Antoni, V.M. Petronis, S. Weiss, R.P. Derhagopian, Optimistic personality and psychosocial well-being during treatment predict psychosocial well-being among long-term survivors of breast cancer, Health Psychol. 24 (5) (2005) 508-516.

[15] M. Chesney, S. Folkman, Psychological impact of HIV disease and implications for intervention, Psychiatr. Clin. N. Am. 17 (1) (1994) 163-182.

[16] M. Chesney, S. Folkman, D. Chambers, Coping effectiveness training for men living with HIV: preliminary findings, Int. J. STD AIDS 7 (Suppl. 2) (1996) 75-82.

[17] M.J. Cordova, L.L. Cunningham, C.R. Carlson, M.A. Andrykowski, Posttraumatic growth following breast cancer: a controlled comparison study, Health Psychol. 20 (3) (2001) 176-185.

[18] J.D. Corrigan, J.A. Bogner, W.J. Mysiw, D. Clincbot, L. Fugate, Life satisfaction after traumatic brain injury, J. Head Trauma Rehabil. 16 (6) (2001) 543-555.

[19] A. Craig, Y. Tran, J. Middleton, Psychological morbidity and spinal cord injury: a systematic review, Spinal Cord 47 (2) (2009) 108-114.

[20] N.M. Crewe, Quality of life-the ultimate goal in rehabilitation, Minn. Med. 63 (8) (1980) 586-589.

[21] J.A. DePalma, P. Fedorka, L.C. Simko, Quality of life experienced by severely in jured trauma survivors, AACN Clin. Issues 14 (1) (2003) 54-63.

[22] E. Diener, E. Suh, Measuring quality of life: economic, social, and subjective indicators, Soc. Indic. Res. 40 (1997) 189-216.

[23] M. Dijkers, Quality of life after spinal cord injury: a meta analysis of the effects of disablement components, Spinal Cord 35 (1997) 829-840. 
[24] M. Dijkers, Quality of life of individuals with spinal cord injury: a review of conceptualization, measurement, and research findings, J. Rehabil. Res. Dev. 42 (3) (2005) 87-110.

[25] D.S. Dunn, Well-being following amputation: salutary effects of positive meaning, optimism, and control, Rehabil. Psychol. 41 (4) (1996) 285-302.

[26] J. Endicott, J. Nee, W. Harrison, R. Blumenthal, Quality of life enjoyment and satisfaction measure: a new measure, Psychopharmacol. Bull. 29 (1993) 321-326.

[27] F. Faul, E. Erdfelder, A. Buchner, A.-G. Lang, Statistical power analyses using $\mathrm{G}^{*}$ power 3.1: tests for correlation and regression analyses, Behav. Res. Methods 41 (2009) 1149-1160.

[28] R.G. Frank, B. Caplan, M. Rosenthal (Eds.), Handbook of Rehabilitation Psychology, 2nd ed., American Psychological Association, Washington, DC, 2010.

[29] N. Garfenski, V. Kraaij, M.J. Schroevers, G.A. Somsen, Post-traumatic growth after a myocardial infarction: a matter of personality, psychological health, or cognitive coping? J. Clin. Psychol. Med. Settings 15 (4) (2008) 270-277.

[30] S.E. Hardy, T.M. Gill, Recovery from disability among community-dwelling older persons, J. Am. Med. Assoc. 291 (2004) 1596-1602.

[31] C.A. Hawley, S. Joseph, Predictors of positive growth after traumatic brain injury: a longitudinal study, Brain Inj. 22 (5) (2008) 427-435.

[32] S.C. Hayes, Acceptance and commitment therapy, relational frame theory, and the third wave of behavioral and cognitive therapies, Behav. Ther. 35 (4) (2004) 639-665.

[33] S.C. Hayes, J.B. Luoma, F.W. Bond, A. Masuda, J. Lillis, Acceptance and commitment therapy: model, processes and outcomes, Behav. Res. Ther. 44 (1) (2006) $1-25$.

[34] G. Henriques, K. Kleinman, C. Asselin, The nested model of well-being: a unified approach, Rev. Gen. Psychol. 18 (1) (2014) 7-18.

[35] T.L. Holbrook, J.P. Anderson, W.J. Sieber, D. Browner, D.B. Hoyt, Outcome after major trauma: 12-month and 18-month follow-up results from the trauma recovery project, J. Trauma 46 (5) (1999) 765-773.

[36] G.N. Holmbeck, Toward terminological, conceptual, and statistical clarity in the study of mediators and moderators: examples from the child-clinical and pediatric psychology literatures, J. Consult. Clin. Psychol. 65 (4) (1997) 599-610.

[37] R. Janoff-Bulman, Shattered Assumptions: Towards a New Psychology of Trauma, Free Press, New York, NY, 1992.

[38] L. Jansen, M. Hoffmeister, J. Chang-Claude, H. Brenner, V. Arndt, Benefit finding and post-traumatic growth in long-term colorectal cancer survivors: prevalence, determinants, and associations with quality of life, Br. J. Cancer 105 (8) (2011) $1158-1165$.

[39] P. Kennedy (Ed.), The Oxford Handbook of Rehabilitation Psychology, Oxford University Press, 2012.

[40] P. Kennedy, A. Kilvert, Coping effectiveness training, Practical Psychology in Medical Rehabilitation, Springer, Cham, 2017, pp. 423-432.

[41] K.B. Kortte, M. Gilbert, P. Gorman, S.T. Wegener, Positive psychological variables in the prediction of life satisfaction after spinal cord injury, Rehabil. Psychol. 55 (1) (2010) 40.

[42] M. Kreuter, A. Siösteen, B. Erkholm, U. Byström, D.J. Brown, Health and quality of life of persons with spinal cord lesion in Australia and Sweden, Spinal Cord 43 (2) (2005) 123-129.

[43] R.A. Kulka, W.E. Schlenger, J.A. Fairbank, R.L. Hough, B.K. Jordan, C.R. Marmar, D.S. Weiss, Trauma and the Vietnam War Generation: Report of Findings From the National Vietnam Veterans Readjustment Study: Brunner/Mazel, (1990).

[44] S.C. Lechner, C. Carver, M. Antoni, K.E. Weaver, Curvilinear associations between benefit finding and psychosocial adjustment to breast cancer, J. Consult. Clin. Psychol. 74 (5) (2006) 828-840.

[45] H. Livneh, Psychosocial adaptation to chronic illness and disability a conceptual framework, Rehabil. Couns. Bull. 44 (3) (2001) 151-160.

[46] H. Livneh, E. Martz, Coping strategies and resources as predictors of psychosocial adaptation among people with spinal cord injury, Rehabil. Psychol. 59 (3) (2014) 329-339.

[47] A. Maercker, T. Zoellner, The Janus face of self-perceived growth: toward a twocomponent model of posttraumatic growth, Psychol. Inq. 15 (1) (2004) 41-48.

[48] D.R. Maki, V.M. Tarvydas, The Professional Practice of Rehabilitation Counseling, Springer Publishing Company, 2011.

[49] E. Martz, T. Bodner, H. Livneh, Coping as a moderator of disability and psychosocial adaptation among Vietnam theater veterans, J. Clin. Psychol. 65 (1) (2009) 94-112.

[50] E. Martz, T. Bodner, H. Livneh, Social support and coping as moderators of perceived disability and posttraumatic stress levels among Vietnam theater veterans, Health 2 (04) (2010) 332-341.

[51] D.L. Miller, S.L. Manne, K. Taylor, J. Keates, J. Dougherty, Psychological distress and well-being in advanced cancer: the effects of optimism and coping, J. Clin. Psychol. Med. Settings 3 (2) (1996) 115-130.

[52] E.F. Morrill, N.T. Brewer, S.C. O'Neill, S.E. Lillie, E.C. Dees, L.A. Carey, B.K. Rimer, The interaction of post-traumatic growth and post-traumatic stress symptoms in predicting depressive symptoms and quality of life, Psycho-Oncology 17 (9) (2008) 948-953.

[53] W.B. Mortenson, L. Noreau, W.C. Miller, The relationship between and predictors of quality of life after spinal cord injury at 3 and 15 months after discharge, Spinal Cord 48 (1) (2010) 73-79.

[54] R. Müller, C. Peter, A. Cieza, S. Geyh, The role of social support and social skills in people with spinal cord injury - a systematic review of the literature, Spinal Cord 50 (2) (2012) 94-106.

[55] C. Nahlén Bose, H. Persson, G. Björling, G. Ljunggren, M.L. Elfström, F. Saboonchi, Evaluation of a coping effectiveness training intervention in patients with chronic heart failure-a randomized controlled trial, Eur. J. Cardiovasc. Nurs. 15 (7) (2016) 537-548.

[56] M.L. O'Donnell, M. Creamer, P. Elliott, C. Atkin, T. Kossmann, Determenants of quality of life and role-related disability after injury: impact of acute psychological responses, J. Trauma 59 (10) (2005) 1328-1335.

[57] K.I. Pakenham, Benefit finding in multiple sclerosis and associations with positive and negative outcomes, Health Psychol. 24 (2) (2005) 123-132.

[58] E.J. Pedhazur, Multiple Regression in Behavioral Research: Explanation and Prediction, Thompson Learning. Inc., New York, NY, 1997.

[59] R.H. Pietrzak, J.M. Cook, Psychological resilience in older US veterans: results from the national health and resilience in veterans study, Depression Anxiety 30 (5) (2013) 432-443.

[60] R.H. Pietrzak, R.B. Goldstein, S.M. Southwick, B.F. Grant, Physical health conditions associated with posttraumatic stress disorder in US older adults: results from wave 2 of the National Epidemiologic Survey on Alcohol and Related Conditions, J. Am. Geriatr. Soc. 60 (2) (2012) 296-303.

[61] M. Post, L. Noreau, Quality of life after spinal cord injury, J. Neurol. Phys. Ther. 29 (3) (2005) 139-146.

[62] T. Powell, A. Ekin-Wood, C. Collin, Post-traumatic growth after head injury: a longterm follow-up, Brain Inj. 21 (1) (2007) 31-38.

[63] T. Powell, R. Gilson, C. Collin, TBI 13 years on: factors associated with post-traumatic growth, Disabil. Rehabil. 34 (17) (2012) 1461-1467.

[64] C. Ramos, P.A. Costa, T. Rudnicki, A.L. Marôco, I. Leal, R. Guimarães, R.G. Tedeschi, The effectiveness of a group intervention to facilitate posttraumatic growth among women with breast cancer, Psycho-Oncology (2017) 1-7.

[65] C. Ramos, I. Leal, R.G. Tedeschi, Protocol for the psychotherapeutic group intervention for facilitating posttraumatic growth in nonmetastatic breast cancer patients, BMC Womens Health 16 (22) (2016) 1-9.

[66] R.E. Renwick, I.E. Brown, M.E. Nagler, Quality of Life in Health Promotion and Rehabilitation: Conceptual Approaches, Issues, and Applications, Sage, Thousand Oaks, 1996.

[67] A.M. Roepke, Psychosocial interventions and posttraumatic growth: a meta-analysis, J. Consult. Clin. Psychol. 83 (1) (2015) 129-142.

[68] P.P. Schnurr, C.A. Lunney, M.J. Bovin, B.P. Marx, Posttraumatic stress disorder and quality of life: extension of findings to veterans of the wars in Iraq and Afghanistan, Clin. Psychol. Rev. 29 (2009) 727-735.

[69] S. Schweininger, D. Forbes, M. Creamer, A.C. McFarlane, D. Silove, R.A. Bryant, M.L. O'Donnell, The temporal relationship between mental health and disability after injury, Depression Anxiety 32 (1) (2015) 64-71.

[70] S.R. Sears, A.L. Stanton, S. Danoff-Burg, The yellow brick road and the emerald city: benefit finding, positive reappraisal coping and posttraumatic growth in women with early-stage breast cancer, Health Psychol. 22 (5) (2003) 487-497.

[71] Z. Solomon, M. Mikulincer, Trajectories of PTSD: a 20-year longitudinal study, Am. J. Psychiatr. 163 (4) (2006) 659-666.

[72] S.A.M. Stevelink, E.M. Malcolm, C. Mason, S. Jenkins, J. Sundin, N.T. Fear, The prevalence of mental health disorders in (ex-) military personnel with a physical impairment: a systematic review, Occup. Environ. Med. 72 (4) (2015) 243-251.

[73] B.G. Tabachnick, L.S. Fidell, Using Multivariate Statistics, 5th edition, Allyn \& Bacon, Needham Height, MA, 2007.

[74] D.G. Tate, M. Forchheimer, C.H. Bombardier, A.W. Heinemann, H.D. Neumann, J.R. Fann, Differences in quality of life outcomes among depressed spinal cord in jury trial participants, Arch. Phys. Med. Rehabil. 96 (2015) 340-348.

[75] S.E. Taylor, D.A. Armor, Positive illusions and coping with adversity, J. Pers. 64 (4) (1996) 873-898.

[76] R.G. Tedeschi, L.G. Calhoun, Trauma and Transformation: Growing in the Aftermath of Suffering, Sage, Thousand Oaks, CA, 1995.

[77] R.G. Tedeschi, L. Calhoun, Posttraumatic growth: a new perspective on psychotraumatology, Psychiatr. Times 21 (4) (2004) 58-60.

[78] R.G. Tedeschi, R.J. McNally, Can we facilitate posttraumatic growth in combat veterans? Am. Psychol. 34 (1) (2011) 19-24.

[79] R.G. Tedeschi, C.L. Park, L.G. Calhoun, Posttraumatic Growth: Positive Changes in the Aftermath of Crisis, Erlbaum, Mahwah, NJ, 1998.

[80] P.L. Tomich, V.S. Helgeson, Is finding something good in the bad always good? Benefit finding among women with breast cancer, Health Psychol. 23 (1) (2004) $16-23$.

[81] J. Tsai, R. El-Gabalawy, W. Sledge, S. Southwick, R. Pietrzak, Post-traumatic growth among veterans in the USA: results from the National Health and Resilience in Veterans Study, Psychol. Med. 45 (01) (2015) 165-179.

[82] J. Tsai, N.P. Mota, S.M. Southwick, R.H. Pietrzak, What doesn't kill you makes you stronger: a national study of U.S. military veterans, J. Affect. Disord. 189 (2016) 269-271.

[83] J. Tsai, L. Sippel, N. Mota, S. Southwick, R. Pietrzak, Longitudinal course of posttraumatic growth among US military veterans: results from the National Health and resilience in veterans study, Depression Anxiety 33 (1) (2016) 9-18.

[84] A.C. Wagner, L. Torbit, T. Jenzer, M.S. Landy, N.D. Pukay-Martin, A. Macdonald, C.M. Monson, The role of posttraumatic growth in a randomized controlled trial of cognitive-behavioral conjoint therapy for PTSD, J. Trauma. Stress. 29 (4) (2016) 379-383.

[85] C.H. Wang, S.L. Tsay, A.E. Bond, Post-traumatic stress disorder, depression, anxiety, and quality of life in patients with traffic-related injuries, J. Adv. Nurs. 52 (1) (2005) 22-30.

[86] F. Weathers, B. Litz, D. Herman, J. Huska, T. Keane, The PTSD Checklist (PCL): Reliability, Validity, and Diagnostic Utility, Paper Presented at the Annual Convention of the International Society for Traumatic Stress Studies, San Antonio, TX, October 1993.

[87] J. Wilson, B. Raphael, International Handbook of Traumatic Stress Syndromes, Springer Science \& Business Media, 2013.

[88] T. Wiseman, K. Foster, K. Curtis, Mental health following traumatic physical injury: an integrative literature review, Injury 44 (11) (2013) 1383-1390.

[89] C. Zhang, E. Leeming, P. Smith, P. Chung, M. Hagger, S. Hayes, Acceptance and commitment therapy for health behavior change: a contextually-driven approach, Front. Psychol. 8 (2018) 1-6. 